\title{
SUBSTITUIÇÃO LOCAL DE AMMOPHILA ARENARIA POR MEDICAGO MARINA EM DUNAS FRONTAIS APÓS PISOTEIO
}

\author{
MARIA MANUELA LARANJEIRA ${ }^{1}$
}

ANA RAMOS-PEREIRA ${ }^{2}$

\begin{abstract}
Resumo - O pisoteio é um dos principais fatores de perturbação da duna frontal, originando uma rede de caminhos e clareiras no meio da vegetação densa, por eliminação de plantas vulneráveis como Ammophila arenaria. Em comunidades vegetais não perturbadas, à medida que $A$. arenaria se torna dominante, a coexistência de outras espécies é inibida, tanto pela sua estratégia de crescimento em falange, como pela diminuição da mobilidade da areia junto à superfície. Porém, a dominância desta perene, responsável pelo crescimento em altura da duna, pode ser localmente invertida após o cessar do pisoteio. Dados recolhidos em três áreas de estudo no litoral de Vila Nova de Gaia em 2006, três anos após a primeira grande intervenção da Câmara Municipal no ordenamento dos acessos à praia através das dunas, sugerem que a súbita abundância de Medicago marina se relacionará com a maior disponibilidade de locais adequados ao seu recrutamento, na sequência da morte de indivíduos de A. arenaria. Ao estabilizar eficazmente a areia de caminhos e clareiras de perturbação, M. marina cria condições para a colonização de variadas espécies anuais e modifica de forma significativa o gradiente de mobilidade e deposição da areia na duna frontal.
\end{abstract}

Palavras-chave: Biogeomorfologia, duna frontal, perturbação, pisoteio, dinâmica de comunidades, filtros ambientais.

Abstract - LOCAL REPLACEMENT OF AMMOPHILA ARENARIA BY MEDICAGO MARINA AFTER FOREDUNE TRAMPLING DisTURBANCE. Human trampling is amongst the major causes of foredune disturbance. It creates a diffuse path network and the opening of bare-ground clearings within dense vegetation patches as a result of plant removal, namely of Ammophila arenaria. In undisturbed plant communities $A$. arenaria becomes dominant, and strongly inhibits the co-existence of other species, due to its phalanx growth strategy

Recebido: Abril 2012. Aceite: Fevereiro 2013.

1 Professora Auxiliar do Departamento de Geografia do Instituto de Ciências Sociais da Universidade do Minho; Investigadora do CEG-IGOT-UL, núcleo SLIF - Sistemas Litorais e Fluviais: Dinâmicas, Mudanças Ambientais e Ordenamento do Território. E-mail: manuela.laranjeira@geografia.uminho.pt

2 Professora Associada do IGOT-UL; Investigadora do CEG-IGOT-UL, núcleo SLIF. E-mail: anarp@campus.ul.pt 
and by decreasing sand mobility near the surface. However, the dominance of this dune-builder may be locally reversed after trampling disturbance. Data gathered in three foredune-sites at Vila Nova de Gaia during 2006, three years after a major intervention by the Municipality to manage beach access through the dunes, suggest that a higher abundance of Medicago marina is linked to an increased availability of recruitment sites, due to death of $A$. arenaria individuals. Acting as a powerful sand stabiliser, M. marina creates favourable conditions for annual species colonization of paths and gaps, significantly changing sand movement and deposition along the foredune gradient.

Keywords: Biogeomorphology, foredunes, disturbance, trampling, community dynamics, environmental filters.

Résumé - REMPLACEMENT LOCAL D'AMMOPHILA ARENARIA PAR MEDICAGO MARINA, APRES PERTURBATION PAR PIETINEMENT DE LA DUNE FRONTALE. Le piétinement humain est une des principales causes de perturbation des dunes frontales. Il est provoqué par le développement de réseaux de pistes et de clairières dans la dense végétation colonisatrice, par élimination des plantes fragiles, comme Ammophila arenaria. Dans les communautés végétales non perturbées, dès que $A$. arenaria devient dominante, elle inhibe la coexistence des autres espèces, en raison de sa stratégie de croissance en phalange et par diminution de la mobilité du sable en surface. Toutefois, la domination de cette plante, responsable de la croissance dunaire en hauteur, peut être localement stoppée par l'arrêt du piétinement. Les données recueillies dans trois zones d'échantillonnage sur le littoral de Vila Nova de Gaia, en 2006, trois ans après une efficace intervention de la municipalité afin d'aménager et de gérer les accès à la plage à travers les dunes, suggèrent que l'abondance soudaine de Medicago marina est liée à la réapparition d'emplacements favorables, à cause de la mort des pieds d'A. arenaria. En agissant comme un stabilisateur puissant du sable des pistes et clairières, M. marina crée les conditions favorables à la colonisation de plusieurs espèces annuelles et modifie de manière significative le gradient de mobilité et de déposition du sable dans la dune frontale.

Mots-clés: Biogéomorphologie, dune frontale, perturbation, piétinement, dynamique des communautés, filtres environnementaux.

\section{INTRODUÇÃO}

\section{Perturbações ambientais e dinâmica das comunidades de plantas}

Considera-se uma perturbação ambiental qualquer fenómeno externo natural ou antrópico, espacial e temporalmente discreto, que altera a estrutura de ecossistemas, comunidades e populações de espécies, afeta a disponibilidade de recursos ou modifica as características físicas do ambiente (Laska, 2001; White e Pickett, 1985). Conceitos amplamente enraizados na teoria ecológica como "equilíbrio único" e "estado normal" perpetuaram a visão de uma "Natureza Benigna", à qual se associa a garantia da recuperação total, o retorno às condições originais ou "normais", uma vez eliminadas as causas de perturbação (de Leo e Levin, 1997). Longe desse pressuposto reconfortante, o paradigma da Ecologia contemporânea assenta no princípio 
de que os sistemas ecológicos se encontram em desequilíbrio, e não em equilíbrio dinâmico (Perry, 2002). Reconhece-se cada vez mais a importância das perturbações ambientais na regulação da estrutura e dinâmica não linear das comunidades ecológicas (Temperton et al., 2004; Jentsch, 2007), o que implica que a sua regeneração conduz a novas condições diferentes das anteriores (Beisner et al., 2003; Jentsch, 2007). Desta maneira, as perturbações assumem um duplo papel, distinguido por White e Jentsch (2004) - por um lado, geram as condições iniciais que desencadeiam a mudança e, por outro, determinam também uma dinâmica continuada no tempo que, mesmo após o seu termo, exerce um controlo decisivo sobre o estabelecimento e a substituição de indivíduos e espécies nas comunidades, definindo assim a(s) trajetória(s) da sucessão ecológica (Jentsch, 2007).

Nos casos em que as perturbações afetam as espécies dominantes (i.e. as mais abundantes) numa comunidade, outras podem aumentar em número e abundância após a sua cessação. Dado que as espécies dominantes inibem a mudança nas comunidades onde se estabelecem, White e Jentsch (2004) sublinham que a sua remoção - ou seja, a remoção da inércia na comunidade - constitui um fator essencial para catalisar a sucessão ecológica; embora isto suceda apenas se as "espécies subordinadas" (sensu Grime, 1998) diferirem das dominantes na sua resposta às condições ambientais causadas pela perturbação. Com efeito, para além de modificarem os filtros ambientais, as perturbações atuam por si só como um filtro sobre as características das espécies, favorecendo e selecionando as que lhes conferem maior capacidade de sobrevivência, reprodução, dispersão e colonização (White e Jentsch, 2004; Jentsch, 2007). Assim, o processo de reagrupamento de espécies num habitat perturbado resulta de uma resposta diferencial, por parte das espécies da comunidade pré-existente e das espécies do "fundo" comum regional, aos efeitos das perturbações ("response rules" sensu Keddy, 1992).

Em comunidades de plantas, as perturbações dão tipicamente origem a espaços abertos de diversas dimensões que, após a sua cessação, podem voltar a ser colonizados (Denslow, 1980, 1985; Łaska, 2001; Walker e del Moral, 2003). De facto, as clareiras criadas na sequência da morte de indivíduos dominantes rapidamente se convertem na sede do recrutamento de outras espécies menos abundantes (Denslow, 1980; Hook et al., 1994; Young et al., 2001; Gardner e Engelhardt, 2008), permitindo inverter localmente o padrão de dominância e diversidade característico de certas comunidades vegetais (Denslow, 1985). Além do acréscimo direto de um fator limitante da maior importância como é o espaço per se para organismos sésseis como as plantas, este tipo de abertura na vegetação está igualmente associado a um aumento da disponibilidade de outros recursos, como a luz incidente e os nutrientes do solo (Denslow, 1985; Krebs, 2001), bem como à modificação de condições físicas, como as temperaturas do ar e do solo, os fluxos do vento e da água (Denslow, 1985). As clareiras de perturbação são, assim, fundamentalmente distintas quanto à sua génese, e consequências ecológicas de outros tipos de clareira, que resultam da natural heterogeneidade na repartição espacial dos recursos. 
Em habitats onde as gramíneas são dominantes, as espécies que primeiro tendem a recolonizar clareiras de perturbação, de um modo espacialmente mais uniforme (não se confinam apenas às margens), são as que na maioria se propagam através de sementes (Kotanen, 1997; Vandvik, 2004). Vandvik (2004) concluiu igualmente que a colonização inicial destas clareiras é limitada pela disponibilidade local em propágulos, verificando-se assim uma tendência geral para que espécies relativamente comuns nas comunidades já estabelecidas se convertam nas colonizadoras de maior sucesso. Não obstante, Clark et al. (2007) demonstraram que a ocorrência e a qualidade de locais disponíveis para o estabelecimento de novos indivíduos suplantam em importância a limitação em sementes na maioria das populações de plantas que analisaram.

Em suma, de acordo com Gardner e Engelhardt (2008), as perturbações ambientais afetam significativamente os três processos, fortemente interligados, em torno dos quais as teorias sobre a composição e diversidade das comunidades de plantas têm revolvido: (i) ocorrência de uma mortalidade espacialmente dependente, que produz a abertura de novos espaços para colonização; (ii) diferentes padrões espaciais de dispersão, que apenas permitem o acesso e a colonização desses novos espaços a um determinado subconjunto de espécies; e, (iii) heterogeneidade local na abundância das espécies, com diferente capacidade de competição, que conduz à gradual, mas inevitável dominância por um número muito limitado de plantas.

\section{Ammophila arenaria, espécie dominante e engenheiro do ecossistema em dunas frontais}

Nos sistemas litorais de duna frontal a mobilidade da areia e o soterramento são reconhecidos fatores de adaptação das plantas (Maun, 1998; Kent et al., 2001; Gilbert et al., 2008) e da distribuição espacial das comunidades vegetais (Moreno-Casasola, 1986; Dech e Maun, 2005). Na realidade, estes fatores atuam como um poderoso filtro ambiental, ao modificarem a estrutura das comunidades de plantas de forma sensível, eliminando seletivamente as espécies intolerantes ao soterramento, reduzindo a abundância das que lhe são menos tolerantes e aumentando a das espécies tolerantes e dependentes (Maun, 1998). Por seu lado, as dunas frontais distinguem-se como sistemas biogeomorfológicos (sensu Stallins, 2002), uma vez que a sua evolução se encontra estreitamente relacionada com a ação directa de um número reduzido de espécies sobre a dinâmica sedimentar eólica, intensificando ou atenuando os efeitos da interferência da topografia dunar sobre a velocidade do vento junto à superfície (Hesp, 2002).

O estímulo positivo da acumulação regular de areia fresca sobre o rápido crescimento vertical da gramínea perene Ammophila arenaria (estorno) e o vigor das suas populações, bem como a extraordinária importância da sua função morfológica por ser diretamente responsável pelo desenvolvimento em altura dos sistemas de duna frontal, são de há muito conhecidos (Ranwell, 1958; Willis et al., 1959), mas continuam a ser objeto de estudo ( Levin et al., 2008). Esta planta assume, assim, um 
papel primordial como engenheiro do ecossistema (Jones et al., 1994, 1997) e como espécie facilitadora (revisão em Bonanomi et al., 2011), criando condições ambientais (habitats) favoráveis ao estabelecimento de diversas espécies - sobretudo as intolerantes ao soterramento - na crista e na vertente a sotavento da duna frontal.

Reunidas as condições ótimas ao seu desenvolvimento, A. arenaria dá origem a povoamentos particularmente densos. Greig-Smith (1961) observou que $A$. arenaria apresenta consistentemente dois tipos de padrão, a duas escalas distintas, ao nível da comunidade: (i) o padrão espacial originado pelos vários rebentos, ou colmos (tillering pattern), que irradiam de um mesmo rizoma vertical, numa escala de 20-40 cm de diâmetro; e, (ii) o padrão espacial formado por uma agregação relativamente densa de diferentes conjuntos de colmos, ou tufo (tussock pattern), numa escala de $80-160 \mathrm{~cm}$ de diâmetro, relacionado com o crescimento de vários rizomas verticais adjacentes, ao longo de um ou mais rizomas horizontais. Pavlick (1983) confirmou experimentalmente que, para $A$. arenaria, o estímulo ao crescimento de rizomas verticais se circunscreve à periferia da planta-mãe, constituindo, por isso, um processo relativamente localizado. O padrão dos colmos atinge densidades máximas onde a deposição de areia em seu redor é moderada (Packham e Willis, 1997) - ou seja, no troço inicial da vertente a sotavento da duna frontal - e, por sua vez, o rápido crescimento vertical de $A$. arenaria é propenso à manutenção do padrão dos tufos (Greig-Smith, 1961), em detrimento da expansão horizontal. Daqui decorre a difusão radial (gradualmente e a curta distância) dos tufos desta planta, que tem como resultado um padrão espacial de crescimento clonal sob a forma de "falange" (Eriksson, 1989), com os novos indivíduos fortemente agregados entre si, em redor da planta-mãe, expandindo-se através de uma frente compacta (phalanx strategy; Doust, 1981). Ora, Doust (1981) propôs que, dada a sua forma de crescimento em falange, as espécies como $A$. arenaria sejam responsáveis pela exclusão de outras plantas do seu "território clonal". Por outro lado, diversos autores têm consistentemente vindo a demonstrar a correlação negativa entre a densidade das espécies perenes dominantes e a distribuição espacial e abundância de outras plantas, em particular as espécies anuais, em dunas litorais (Pemadasa et al., 1974; Watkinson, 1990; Cheplick, 2005). Estas últimas ocorrem, sobretudo, a partir da crista da duna para o interior e, em geral, só conseguem vingar onde as espécies perenes são menos abundantes e mais esparsas.

Com o aumento da cobertura vegetal tem início o processo de estabilização da areia nas dunas (Moreno-Casasola, 1986). Watkinson (1990) concluiu que o declínio na abundância da espécie anual Vulpia fasciculata, ao longo de um período de 9 anos, teve precisamente como fator principal a acentuada diminuição de areia descoberta e móvel, em resultado do aumento da abundância de espécies perenes. Com efeito, o autor provou que este aumento afetou consideravelmente a mortalidade dos rebentos de $V$. fasciculata, dada a germinação das suas sementes à superfície, onde a areia se encontra mais estabilizada e compactada pelas plantas perenes. Da mesma forma, à medida que $A$. arenaria se torna mais abundante e os seus tufos se adensam, tanto a mobilidade da areia junto à superfície como a respetiva quantidade 
a descoberto sofrem uma redução significativa. Nestas condições, a sua dominância poderá regular a presença e a abundância das demais espécies. Assim sendo, à medida que $A$. arenaria se estabelece como a espécie dominante na duna frontal, o seu papel facilitador inicial parece ser substituído pela forte inibição da coexistência de outras plantas, sugerindo que se assiste ao duplo efeito (positivo e negativo) dos engenheiros do ecossistema sobre a diversidade e a abundância das restantes espécies da comunidade, aspeto discutido por Jones et al. (1997).

\section{Objetivos da investigação}

Neste trabalho, procurou-se estabelecer de que forma a estrutura (i.e. composição em espécies e respetiva abundância) das comunidades de plantas em sistemas de duna frontal é modificada e se reconstitui, após anos de perturbação ambiental associada ao pisoteio desregrado no acesso à praia, responsável pela fragmentação dos habitats dunares e a eliminação parcial de A. arenaria (Laranjeira, 2010). Assim sendo, as questões a que se pretendeu dar resposta foram as seguintes: (i) é possível identificar mudanças na estrutura das comunidades de plantas dunares perturbadas pelo pisoteio? (ii) que espécies discriminam estas comunidades perturbadas? (iii) verifica-se uma inversão local da dominância de $A$. arenaria nestas comunidades? e, por fim, (iv) tendo presente a estreita interdependência entre a vegetação, a mobilidade da areia e a morfologia nas dunas frontais, quais as consequências biogeomorfológicas decorrentes de uma substituição local de $A$. arenaria, durante a fase inicial de regeneração destes sistemas?

\section{II. ÁREA DE ESTUDO}

O trabalho foi conduzido em três setores do sistema de duna frontal no litoral de Vila Nova de Gaia, situado no NW Atlântico de Portugal, região cujas comunidades de plantas dunares foram, nos seus aspetos gerais, caracterizadas em Lomba et al. (2008). Trata-se de um litoral baixo, predominantemente arenoso, em que as praias raramente ultrapassam $100 \mathrm{~m}$ de largura e onde existem afloramentos rochosos no meio da praia e a pontuar a linha de costa, o que lhe confere, no pormenor, um traçado ondulado. Os afloramentos rochosos e a largura da praia evidenciam a pequena espessura dos sedimentos arenosos nas praias expostas e predominantemente dissipativas. Refira-se também que este troço litoral se situa a sotamar de uma obra de defesa costeira destacada $125 \mathrm{~m}$. As praias são atravessadas por pequenos cursos de água, muitos dos quais completamente artificializados sob os núcleos urbanos costeiros, cuja foz é desviada para sul, evidenciando claramente a deriva litoral predominante de $\mathrm{N}$ para $\mathrm{S}$, na sequência da incidência oblíqua da ondulação dominante de NW (Ramos-Pereira 2004, Ramos-Pereira et al., 2006; fig. 1).

O litoral em análise encontra-se em erosão há pelo menos meio século, tendência que se iniciou ou intensificou na década de 1970, vinte anos após o início da fase 
de construção de barragens, nomeadamente no Rio Douro, maior abastecedor de sedimentos e cuja foz se situa a barlamar. Acresce, ainda, que os sedimentos dragados no estuário deste rio parecem atingir o volume estimado de sedimentos em trânsito na deriva litoral (Gomes et al., 2006). A escassez em sedimentos resultante da diminuição do seu afluxo à linha de costa, devido ao ordenamento das bacias hidrográficas, assim como a ocupação indevida de sistemas dunares subtraem areias ao litoral, num quadro de subida centenária do nível do mar.

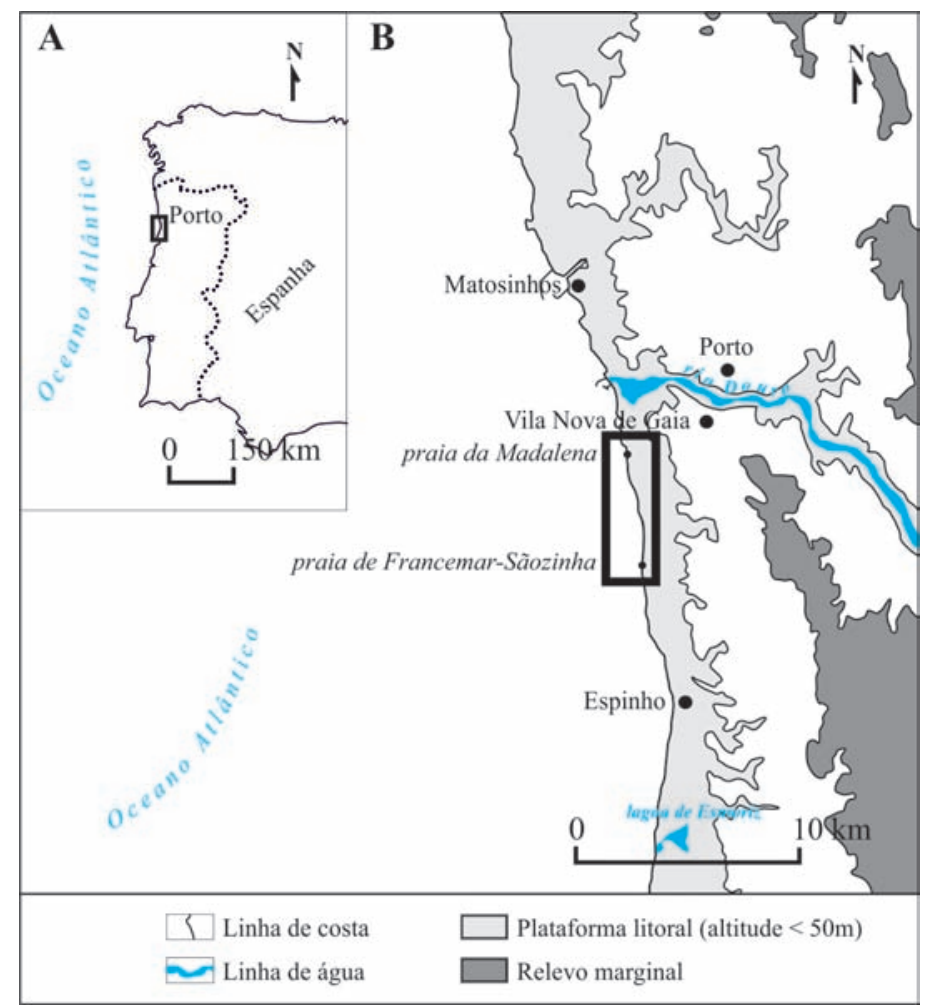

Fig. 1 - O troço litoral estudado. O retângulo vertical corresponde à imagem inferior da fig. 2. Fig. 1 - The studied stretch of coast. The vertical rectangle corresponds to the bottom image in fig. 2.

Entre 2002 e 2003, o litoral estudado foi objeto de uma intervenção de conjunto pela Câmara Municipal de Vila Nova de Gaia, no âmbito do Plano de Ordenamento da Orla Costeira (POOC) em vigor que, entre outras estratégias, procurou ordenar o acesso à praia, através da duna frontal, com: (i) a instalação de vários passadiços sobrelevados, perpendiculares ao cordão dunar, na entrada de cada praia do concelho; (ii) a instalação de um passadiço contínuo, com cerca de $15 \mathrm{~km}$ (sensivelmente a extensão da linha de costa do concelho, entre a foz do Douro e o limite administrativo com o município de Espinho), que marca a atual base da vertente a barlavento; e, (iii) a colocação de armadilhas de areia (estacaria), ao longo de toda a frente dunar. 
As três áreas do sistema dunar frontal, que foram investigadas, caracterizam-se por uma superfície total e proporção da cobertura vegetal comparáveis $\left(\approx 3000-5000 \mathrm{~m}^{2}\right.$ e 30-40\%, respetivamente), embora se diferenciem no grau de fragmentação dos habitats como resultado do pisoteio (Laranjeira, 2010). Duas delas localizam-se na praia da Madalena, separadas entre si por uma brecha bem definida; a terceira diz respeito ao troço entre as praias de Francemar e Sãozinha (fig. 2). A seleção destas áreas obedeceu a um conjunto de critérios, nomeadamente: (i) o gradiente ambiental do cordão dunar frontal ser reconhecível, apesar de fragmentado pelo pisoteio (excluíram-se do estudo as comunidades da praia alta, em geral ausentes devido à erosão marinha e à ocupação por infraestruturas e equipamentos de apoio à atividade balnear, e as comunidades do corredor interdunar, parcialmente urbanizado); (ii) a influência da dinâmica erosiva (sapamento pelo mar e/ou deflação) não se sobrepor à da perturbação pelo pisoteio no que respeita a persistência e distribuição espacial das espécies e comunidades de plantas.

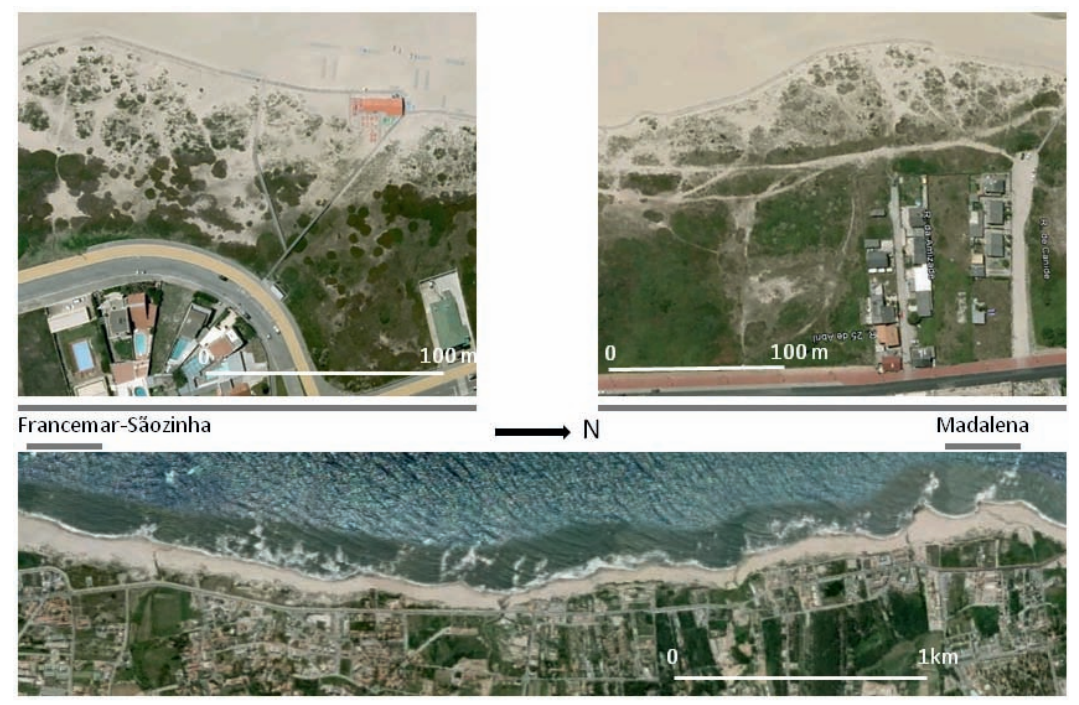

Fig. 2 - Localização das três áreas de estudo no litoral de Vila Nova de Gaia.

Fig. 2 - Location of the three study areas in Vila Nova de Gaia coast.

(See coloured version online)

\section{MÉTODOS UTILIZADOS}

\section{Amostragem de campo e dados sobre a vegetação}

As manchas (ou fragmentos) remanescentes de vegetação dunar, que resultaram da fragmentação pelo pisoteio, foram delimitadas a partir de ortofotos digitais datados de 2000, com uma resolução de $25 \mathrm{~cm}$. Vários autores demonstraram a forte 
correlação existente entre a mobilidade da areia, a topografia dunar e a distribuição espacial das comunidades de plantas (Moreno-Casasola, 1986; Dech e Maun, 2005; Levin et al., 2008; Honrado et al., 2010). Acosta et al. (2007) postularam que o declive e a orientação da superfície dunar constituem duas variáveis essenciais para a compreensão da relação entre o zonamento dos principais tipos de comunidades de plantas e a morfologia dunar local. Nesta sequência, Laranjeira (2010) propôs uma tipologia dos habitats da duna frontal em função da sua posição topográfica. Através de levantamentos de campo de pormenor, realizados em 2006, validou-se a delimitação realizada previamente com base nos ortofotos e classificaram-se todos os fragmentos de vegetação com base nessa tipologia, que inclui: (i) o habitat da vertente a barlavento; (ii) o habitat da crista da duna; (iii) o habitat da vertente a sotavento; e, (iv) as combinações possíveis entre estes habitats, num mesmo fragmento.

O levantamento de dados sobre a estrutura das comunidades de plantas teve lugar entre junho e setembro de 2006. Selecionou-se o método da amostragem aleatória através de quadrados (random quadrating; Reid e Thompson, 1996), com a dimensão de $1 \mathrm{~m}^{2}$ (Smith e Smith, 2001), que foi aplicado a todos os fragmentos com uma superfície $>20 \mathrm{~m}^{2}$, abrangendo as diversas classes de habitat dunar identificadas e distribuídos pelas três áreas de estudo. Reid e Thompson (1996) recomendam que, ao utilizar quadrados, o tamanho da amostra abranja 5\% da área de vegetação em causa (no caso presente, $5 \%$ de cada fragmento); nesta sequência, levantou-se um total de 126 quadrados, para um conjunto de 52 fragmentos com dimensões entre 20 e $245 \mathrm{~m}^{2}$. Ainda que no essencial a disposição dos quadrados em cada fragmento amostrado tenha sido aleatória, houve, contudo, a necessidade de impor duas condições (fig. 3): (i) não permitir a sobreposição parcial dos quadrados; e, (ii) só levantar os quadrados localizados a uma distância superior a $1 \mathrm{~m}$ do perímetro da mancha, a fim de evitar uma possível influência dos efeitos de orla nos dados amostrados.

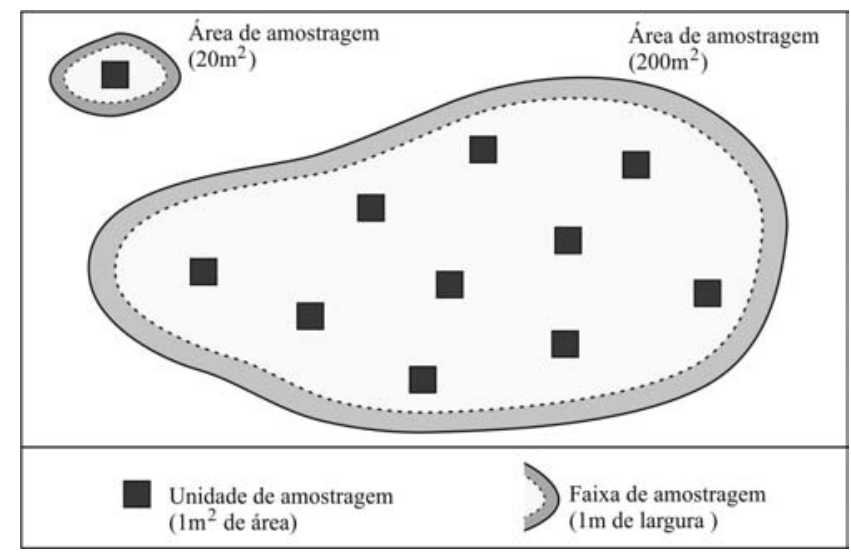

Fig. 3 - Ilustração do esquema de amostragem de campo. Fig. 3 - Field sampling scheme. 
Finalmente, registou-se a presença de todas as espécies em cada quadrado e estimou-se, de forma expedita, a abundância de cada uma delas, através de um sistema de classes. A informação recolhida no campo foi posteriormente convertida no valor central da classe (quadro I).

Quadro I - Estimativa da abundância das espécies presentes nos quadrados. Table I-Estimation of abundance of species present in sampling plots.

\begin{tabular}{cc}
\hline $\begin{array}{c}\text { Classe de abundância } \\
\left(\mathrm{n}^{\circ} \text { indivíduos }\right)\end{array}$ & $\begin{array}{c}\text { Valor central da classe } \\
\left(\mathrm{n}^{\circ} \text { indivíduos }\right)\end{array}$ \\
\hline $\mathbf{1}=1$ & 1 \\
$\mathbf{2}=2$ a 5 & 4 \\
$\mathbf{3}=6$ a 10 & 8 \\
$\mathbf{4}=11$ a 15 & 13 \\
$\mathbf{5}=16$ a 20 & 18 \\
$\mathbf{6}=21$ a 30 & 26 \\
$7=31$ a 40 & 36 \\
\hline
\end{tabular}

Nos fragmentos com uma área $\geq 40 \mathrm{~m}^{2}$, os dados sobre a abundância de cada espécie foram coligidos a partir de 2 ou mais quadrados (ou seja, 2 ou mais subamostras). A fim de evitar um eventual problema de pseudoreplicação, devida à autocorrelação espacial, este conjunto de dados deve ser sintetizado num único valor, que caracterize o fragmento de vegetação como um todo (Feinsinger, 2001). Neste estudo, verificou-se um grande número de valores nulos (ausência de indivíduos) nas subamostras de cada fragmento, visto que raramente os indivíduos das espécies em causa se distribuem de uma forma regular no espaço; assim, calculou-se a média para o conjunto destes dados, em detrimento de outras medidas de tendência central como a mediana ou a moda. Desta maneira, analisaram-se séries de dados da riqueza específica (número total de espécies presentes) e da abundância média (número médio estimado de indivíduos $/ \mathrm{m}^{2}$ ) de cada espécie identificada, para os 52 fragmentos amostrados. Das 21 espécies registadas, excluíram-se da investigação seis, presentes em menos de $6 \%$ das amostras.

\section{Tratamento estatístico dos dados}

Em primeiro lugar, aferiu-se a relação existente entre a abundância média das espécies e a riqueza específica ( $\mathrm{N}^{\mathrm{o}}$ ESPÉCIES) nos fragmentos, bem como entre a abundância média de $A$. arenaria e a de cada uma das restantes 14 plantas. Para tal, utilizou-se o teste de correlação ordinal de Spearman (Townend, 2002), que constitui a alternativa não-paramétrica à correlação de Pearson (correlação linear). A correlação de Spearman avalia até que ponto a ordem dos valores (e não os valores per se) nas séries de duas variáveis exibe uma associação perfeitamente monótona. Quando a ordem de todos os valores da variável $x$ se ajusta rigorosamente à ordem de todos os valores da variável $y$, tem-se uma associação perfeitamente monótona e positiva 
e o coeficiente $\rho=1$; o inverso sucede $\operatorname{com} \rho=-1$. Desta maneira, $\rho$ varia entre -1 e 1 (associações perfeitas), indicando $\rho=0$ uma relação totalmente aleatória entre os números de ordem das duas variáveis em causa.

De seguida, analisou-se a similaridade da estrutura das 52 comunidades de plantas amostradas através do método de classificação hierárquica, politético e divisivo, TWINSPAN (Two-Way Indicator Species Analysis; Hill, 1979). Uma das principais vantagens deste método é a de incorporar a determinação de espécies indicadoras, que caracterizam e diferenciam entre si os grupos de amostras (Dale, 1995). Outra característica do TWINSPAN é a de que assenta na avaliação de padrões de presença/ausência - ou seja, não examina os valores per se da abundância das espécies em cada amostra. Na realidade, este método socorre-se da pré-definição de classes de abundância - designadas de pseudoespécies - para analisar indirectamente aqueles valores, sob a forma da sua presença/ausência em cada uma destas classes (Vermeersch et al., 2003).

O resultado final do TWINSPAN é sintetizado num dendrograma da classificação das amostras por grupos similares, com a identificação das espécies indicadoras inerentes a cada dicotomia. Além disso, os grupos são dispostos de maneira a que se encontrem lado a lado pela sua semelhança, formando portanto uma sequência ordenada, que pode revelar os gradientes ambientais que determinam a variação da estrutura das comunidades de plantas (Gauch e Whittaker, 1981).

A força, ou intensidade, da relação de similaridade entre a estrutura das comunidades de plantas agrupadas através do método TWINSPAN foi avaliada com recurso ao teste de Mantel (Fortin et al., 2002; Urban, 2003). Este teste avalia a intensidade da correlação entre duas matrizes, cujos elementos correspondem a uma medida de similaridade (ou distância) calculada para todas as combinações possíveis entre os dados de duas variáveis (Urban, 2003). Assim, procedeu-se ao teste da correlação entre as séries da abundância média das 15 espécies analisadas (matriz principal de dados originais) e dois tipos de agrupamentos resultantes da classificação TWINSPAN (matrizes secundárias de dados originais): (i) os grupos de amostras diretamente obtidos através do método, designados por 'Grupos TWINSPAN'; e, (ii) os grupos derivados dos anteriores em função das espécies indicadoras com maior abundância, designados por 'Grupos ESPÉCIES DISCRIMINANTES'.

\section{RESULTADOS}

\section{Riqueza específica e abundância das espécies}

O quadro II sintetiza os principais resultados dos testes de correlação de Spearman. Em primeiro lugar, sobressai o fato da abundância das plantas da duna frontal estudadas, à exceção de $A$. arenaria e de $A$. crithmifolia, tender a ser superior nos fragmentos de vegetação com maior diversidade de espécies. Com efeito, $\mathrm{N}^{\circ}$ ESPÉCIES explica quase $40 \%$ da variação da abundância média de E. mari- 
timum, mais de $20 \%$ desta variação para A. bulbosa, L. polygalifolia e E. paralias, e entre $18 \%$ e $9 \%$ nos casos de P. maritimum, V. alopecurus, C. edulis, C. maritima, L. ovatus e E. farctus (embora estatisticamente não significativo, o sentido da associação entre estas 2 variáveis também é positivo para L. taraxacoides, M. marina e C. soldanella). Em segundo lugar, atente-se nas correlações negativas significativas entre a abundância média de M. marina, E. farctus e A. crithmifolia e a de A. arenaria.

Quadro II - Coeficientes $\rho$ das correlações significativas $(p \leq 0,05)$ segundo o teste de Spearman, e respetivos coeficientes de determinação $\rho^{2}(\%)$, entre o número de espécies, a abundância média de A. arenaria e a das restantes espécies estudadas (entre parêntesis, indica-se o sentido das correlações não significativas).

Table II - Significant $(p \leq 0,05)$ Spearman rank correlation coefficients $(\rho)$, and respective $\rho^{2}$ value $(\%)$, between species number, average abundance of $A$. arenaria and average abundance of all other studied species (in brackets, the \pm sign refers to the nature of non-significant correlations).

\begin{tabular}{|c|c|c|c|}
\hline & Espécie & $\begin{array}{l}\text { Abundância média } \\
\text { Ammophila arenaria }\end{array}$ & $\mathrm{N}^{\mathrm{o}}$ espécies \\
\hline \multirow{15}{*}{ 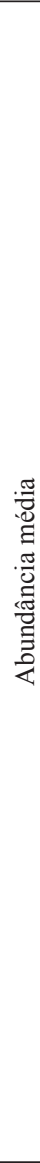 } & Eryngium maritimum (Cardo-marítimo) & $(+)$ & $\begin{array}{l}+0,627 \\
\rho^{2}=39,3\end{array}$ \\
\hline & Pancratium maritimum (Lírio-das-areias) & $(+)$ & $\begin{array}{l}+0,423 \\
\rho^{2}=17,9\end{array}$ \\
\hline & Aetheorhiza bulbosa (Condrilha-de-Dioscórides) & $(-)$ & $\begin{array}{l}+0,517 \\
\rho^{2}=26,7\end{array}$ \\
\hline & Leontodon taraxacoides (Leituga-dos-montes) & $(-)$ & $(+)$ \\
\hline & Medicago marina (Luzerna-das-praias) & $\begin{array}{c}-0,337 \\
\rho^{2}=11,4\end{array}$ & $(+)$ \\
\hline & Carpobrotus edulis (Chorão) & $(-)$ & $\begin{array}{l}+0,347 \\
\rho^{2}=12,0\end{array}$ \\
\hline & Euphorbia paralias (Morganheira-das-praias) & $(+)$ & $\begin{array}{l}+0,466 \\
\rho^{2}=21,7\end{array}$ \\
\hline & Elymus farctus (Feno-das-areias) & $\begin{array}{c}-0,387 \\
\rho^{2}=15,0\end{array}$ & $\begin{array}{l}+0,301 \\
\rho^{2}=9,0\end{array}$ \\
\hline & Calystegia soldanella (Couve-marinha) & $\begin{array}{l}+0,404 \\
\rho^{2}=16,3\end{array}$ & $(+)$ \\
\hline & Crucianella maritima (Granza-das-praias) & $(+)$ & $\begin{array}{l}+0,322 \\
\rho^{2}=10,3\end{array}$ \\
\hline & Linaria polygalifolia (Ansarina) & $(-)$ & $\begin{array}{l}+0,482 \\
\rho^{2}=23,2\end{array}$ \\
\hline & Vulpia alopecurus (Vúlpia) & $(-)$ & $\begin{array}{l}+0,370 \\
\rho^{2}=13,7\end{array}$ \\
\hline & Lagurus ovatus (Rabo-de-lebre) & $(-)$ & $\begin{array}{l}+0,312 \\
\rho^{2}=9,7\end{array}$ \\
\hline & Artemisia crithmifolia (Madorneira) & $\begin{array}{c}-0,466 \\
\rho^{2}=21,7\end{array}$ & $(-)$ \\
\hline & Ammophila arenaria (Estorno) & - & $(-)$ \\
\hline
\end{tabular}




\section{Grupos de amostras e espécies discriminantes}

Através da análise dos resultados da classificação TWINSPAN, procurou-se estabelecer o significado ecológico dos diversos grupos de amostras obtidos (grupos de comunidades similares de plantas), bem como o da sua sequência ordenada. Esta classificação separa perfeitamente, desde a primeira divisão, as amostras onde A. crithmifolia está ou não presente (fig. 4). Os grupos das comunidades (Grupos 8 a 10) em que esta espécie subarbustiva é a dominante (pseudoespécie 5) surgem no extremo de um primeiro gradiente, que parece estar grosso modo relacionado com a variação do habitat ao longo da duna frontal.

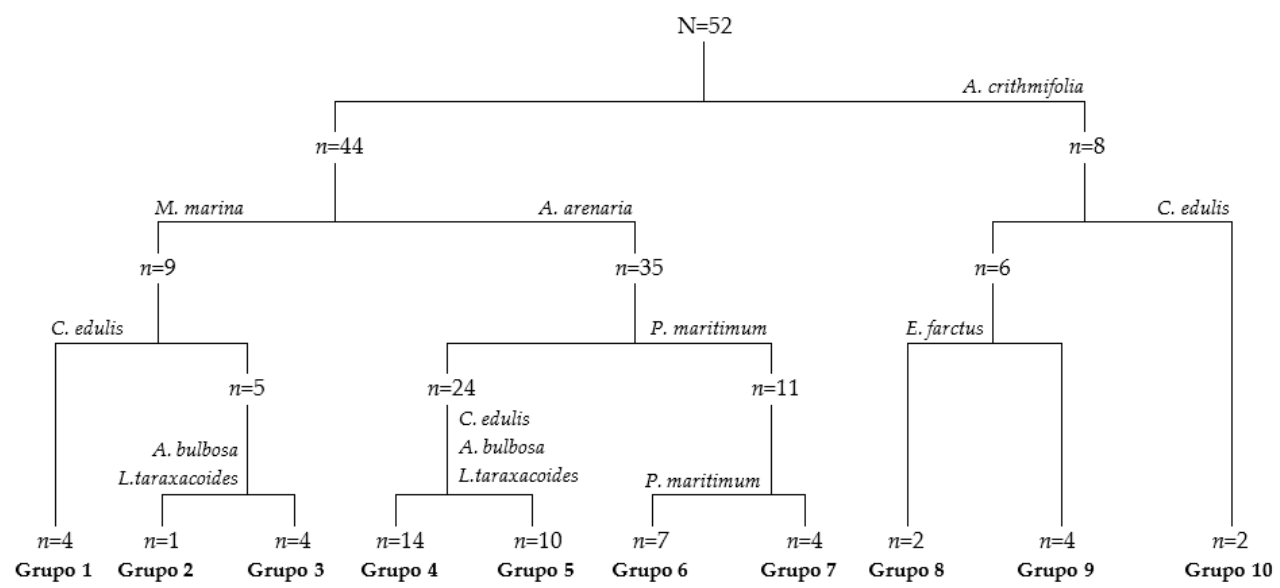

Fig. 4 - Dendrograma da classificação TWINSPAN das comunidades de plantas amostradas, até ao $4^{\circ}$ nível de divisão ( $\mathrm{N}=$ número total de amostras; $\mathrm{n}=$ número de amostras em cada lado da dicotomia). Fig. 4 -Dendrogram of TWINSPAN classification of sampled plant communities, to the $4^{\text {th }}$ cut level ( $N=$ total number of samples; $n=$ number of samples at each side of the dichotomy).

O $2^{\circ}$ nível de divisão, no que toca ao grupo negativo obtido na dicotomia anterior $(n=44)$, baseia-se nas espécies mais abundantes das diversas amostras para as separar entre si. Assim, 67,3\% $(n=35)$ das comunidades foram classificadas pela dominância de $A$. arenaria (pseudoespécie 5) e 17,3\% $(n=9)$ pela relativa abundância de M. marina (pseudoespécie 3), (fig. 4). Para as subsequentes divisões da maioria dos grupos, o critério da presença/ausência prevaleceu sobre o da abundância na definição das sucessivas espécies indicadoras, estando a pseudoespécie 1 quase sempre associada a estas. Não deixa de ser notável que as espécies indicadoras definidas nos $2^{\circ}$ e $3^{\circ}$ níveis de divisão, para os Grupos 8 a 10 , e entre os $3^{\circ}$ e $4^{\circ}$ níveis de divisão, para os Grupos 1 a 5, sejam C. edulis, E. farctus, A. bulbosa e L. taraxacoides (fig. 4).

Atendendo a estes resultados, o grau de similaridade entre a estrutura das comunidades de plantas foi aferido para: (i) os 10 grupos obtidos até ao $4^{\circ}$ nível de divisão (Grupos TWINSPAN); e (ii) os 3 grupos diferenciados pelas espécies indica- 
doras com a maior abundância (Grupos ESPÉCIES DISCRIMINANTES), que correspondem a $A$. arenaria (Aa; pseudoespécie 5; Grupos 4 a 7), A. crithmifolia (Ac; pseudoespécie 5; Grupos 8 a 10) e M. marina (Mm; pseudoespécie 3; Grupos 1 a 3), (fig. 4 e quadro III). Em ambos os casos, os testes de Mantel confirmaram a elevada similaridade entre o padrão de presença e abundância das espécies nos diversos fragmentos de vegetação que pertencem a um mesmo grupo (como também a sua inequívoca dissimilaridade entre fragmentos de grupos diferentes; quadro III). Assim, é possível distinguir claramente entre as comunidades dominadas por A. arenaria, A. crithmifolia e M. marina.

Quadro III - Coeficientes de correlação $r$ dos testes de Mantel (e respetivo $p$-valor) entre a abundância média das espécies e grupos de comunidades de plantas amostradas similares.

Table III - Mantel test correlation coefficients ( $r$ ), and respective p-value, between species average abundance and groups of similar sampled plant communities.

\begin{tabular}{cccc}
\hline $\begin{array}{c}\text { Variáveis dependentes } \\
\text { (Matriz Principal) }\end{array}$ & $\begin{array}{c}\text { Variáveis independentes } \\
\text { (Matriz Secundária) }\end{array}$ & $r$ & $p$-valor \\
\hline $\begin{array}{c}\text { Abundância média } \\
\text { (conjunto das 15 espécies) }\end{array}$ & Grupos TWINSPAN & 0,60 & $<0,0001$ \\
\cline { 2 - 4 } & $\begin{array}{c}\text { Grupos ESPÉCIES DISCRIMINANTES } \\
\text { (Aa, Ac e Mm) }\end{array}$ & 0,64 & $<0,0001$ \\
\hline
\end{tabular}

\section{DISCUSSÃO}

Os resultados obtidos com a correlação de Spearman apontam para a evidência de que $A$. arenaria, enquanto dominante, inibe a coexistência de outras espécies tanto em número como em abundância, tal como Honrado et al. (2010) concluíram para dunas frontais, que manifestam uma dinâmica transgressiva no litoral NW português. Além disso, sugerem que a mobilidade da areia, variável diretamente modificada por esta planta, constitui um fator primordial à escala local para manter a presença de toda uma série de espécies anuais e vivazes (Forey et al., 2008), e assegurar a sua diversidade máxima nas dunas frontais. Essas conclusões podem avaliar-se no quadro II:

- na vertente a barlavento, onde o stress ambiental é intenso devido à elevada mobilidade da areia, ao predomínio da deflação sobre a deposição e à forte salinidade, E. farctus é mais abundante em detrimento de A. arenaria;

- junto à crista da duna, onde a presença de $A$. arenaria estimula a acumulação, mas a percentagem de areia móvel e a descoberto se mantém elevada, espécies como C. soldanella tendem a beneficiar das condições geomorfológicas criadas por esta gramínea;

- ainda no topo da duna, mas já a sotavento, os tufos de $A$. arenaria adensam-se e, por consequência, tanto a cobertura vegetal como a estabilização da areia junto à superfície aumentam significativamente; onde se formam povoamentos muito agregados, estas condições agravam-se, limitando a presença ou a abundância de certas espécies que ocorrem neste habitat, como M. marina. 
No que respeita a correlação negativa entre a abundância média de $A$. arenaria e a de $A$. crithmifolia, e correspondendo estas às duas únicas espécies naturais dos sistemas de duna frontal estudados, que se tornam dominantes nos respetivos habitats ótimos - caso da crista da duna para a primeira e da vertente a sotavento para a segunda (Lomba et al., 2008) -, ambas dão origem a povoamentos particularmente densos, que excluem a presença uma da outra, ou limitam drasticamente o seu vigor. Com efeito, também no caso do subarbusto $A$. crithmifolia, as indicações constantes da base de dados 'Ecological Flora of the British Isles', da Universidade de York, sobre a sua reprodução vegetativa, mais concretamente o método - rhizome shortly creeping - e o padrão - patch-forming -, são consistentes com a forma de crescimento clonal em falange.

O destaque que M. marina merece logo no início do processo divisivo TWINSPAN, por oposição a $A$. arenaria (fig. 4), é bastante revelador. Na realidade, este resultado sugere que a sua maior abundância em certos fragmentos de habitat da duna frontal, em detrimento da de $A$. arenaria, é indicadora de perturbação das respetivas comunidades de plantas. Com efeito, tendo em conta a correlação negativa entre a abundância média destas 2 espécies (quadro II) e o fato das comunidades dominadas por M. marina serem suficientemente distintas das restantes (quadro III), é plausível assumir que esta planta constitui povoamentos mais expressivos em fragmentos próximos à crista da duna, onde a vegetação original terá sofrido maior perturbação devido ao pisoteio desregrado, nomeadamente com a eliminação parcial de A. arenaria e a subsequente abertura de clareiras. Na investigação desenvolvida por Honrado et al. (2010), de entre as 9 espécies mais frequentes nas dunas frontais analisadas, M. Marina é a única cuja ocorrência regista um franco incremento em condições transgressivas. À luz dos resultados do presente trabalho, este dado tão interessante levanta a hipótese de que tal aumento de M. marina não se deverá exclusivamente, ou diretamente, à dinâmica transgressiva das dunas, mas ao efeito sinergístico desta com o da perturbação relacionada com o pisoteio, comum no litoral NW. Daqui decorre que a ocorrência discriminante desta espécie nas comunidades de plantas estudadas por aqueles autores poderá estar relacionada com fatores à escala local, e não com variações biogeográficas, à escala regional (Honrado et al., 2010).

Para além de $M$. marina, a classificação TWINSPAN faz sobressair como espécies indicadoras outras plantas que denunciam condições de perturbação das comunidades que caracterizam, nomeadamente (fig. 4): (i) C. edulis (Grupos 1, 5 e 10) é uma espécie exótica listada como invasora no Decreto-Lei 565/99, de 21 de dezembro; (ii) E. farctus (Grupo 8) surge numa posição atípica ou "secundária" (Araújo et $a l ., 2002$ ), em consequência da acumulação a sotavento das areias remobilizadas ao longo dos principais caminhos/corredores eólicos da duna frontal; e, (iii) $A$. bulbosa e L. taraxacoides (Grupos 2 e 5) são espécies que ocorrem, com frequência, em locais ruderalizados. Resultados idênticos a estes, obtidos a partir da classificação de amostras da estrutura da vegetação pelo método TWINSPAN, foram descritos para sistemas dunares muito degradados pelo pisoteio, no litoral ocidental de Creta (Tzatzanis et al., 2003). 
O dendrograma da figura 4 sugere que, ao $4^{\circ}$ nível de divisão, a sequência ordenada dos grupos de comunidades similares de plantas tende a esboçar um gradiente secundário de perturbação ambiental, que vem introduzir alterações a uma primeira ordenação e posicionamento relativo destes grupos ao longo de um gradiente principal correspondente à variação dos habitats da duna frontal - desde os habitats da vertente a barlavento e crista (Grupos 4 a 7) até ao habitat da vertente a sotavento (Grupos 8 a 10). Sendo assim, é possível ajuizar que as manchas mais perturbadas (e onde a estrutura da comunidade de plantas se encontra mais modificada) serão as pertencentes aos Grupos 1 a 3, onde a danificação e eliminação de A. arenaria terá favorecido a maior abundância de M. marina.

\section{CONCLUSÃO}

\section{Efeito de filtro ambiental por Medicago marina}

Como consequência do pisoteio, a dominância característica de A. arenaria na duna frontal pode sofrer localmente uma inversão. A classificação pelo método TWINSPAN das comunidades de plantas estudadas discriminou M. marina como uma das principais espécies indicadoras dos grupos obtidos. A presente investigação aponta para o fato deste resultado - associado à maior abundância de M. marina nas comunidades que permite diferenciar - se relacionar com a rápida colonização, por parte desta espécie, de pequenas clareiras abertas na sequência da morte de indivíduos de $A$. arenaria pelo pisoteio (menos assíduo e intenso que nos caminhos). A evidência de uma substituição local da dominante $A$. arenaria por $M$. marina sai reforçada pelo resultado da correlação negativa entre a abundância média de ambas as espécies. Assim sendo, a estrutura das comunidades de plantas, nos habitats dunares fragmentados pelo pisoteio, é sensivelmente modificada devido ao aparecimento de clareiras de perturbação.

Em consonância com o modelo de Keddy (1992), os resultados obtidos sugerem que, de entre as espécies do "fundo" comum regional, M. marina sobressai como aquela que reúne as melhores caraterísticas para beneficiar das novas condições ambientais nas clareiras de perturbação. Com efeito, trata-se de uma espécie que, simultaneamente:

- se dispersa através de sementes e, apesar de surgir com maior frequência próximo da crista da duna, é relativamente comum ao longo de todo o gradiente ambiental da duna frontal, o que lhe confere uma potencial disponibilidade de propágulos bastante elevada;

- beneficia do aumento da luz direta (espécie pioneira, que não suporta a sombra; Bonanomi et al., 2008) e da movimentação superficial da areia (suporta o soterramento e retém as areias; grupo funcional Type III, de acordo com García-Mora et al., 1999), resultantes da ausência de tufos de $A$. arenaria, o que eleva significativamente as probabilidades de germinação e sobrevivência das plântulas nas clareiras de perturbação, por comparação com o que sucede no meio da vegetação densa. 
Já que a disponibilidade em sementes não constitui uma limitação ao recrutamento de M. marina, uma vez suprimida a restrição em microhabitats adequados ao seu desenvolvimento ótimo com a morte de indivíduos da espécie dominante, aquela passa a reunir as duas condições que, segundo Eriksson e Ehrlén (1992, incrementam o estabelecimento de plântulas, promovendo uma rápida propagação. Por estas razões, embora as espécies anuais termófilas dependam da existência de pequenos espaços abertos no meio da vegetação densa para vigorar, o facto de serem plantas incapazes de sobreviver ao soterramento pela areia faz com que não colham inicialmente nenhuma vantagem das clareiras originadas pelo pisoteio nos habitats próximos da crista da duna, tal como sucede com M. marina.

Em suma, argumenta-se que $M$. marina exerce um papel fulcral, nas primeiras fases de regeneração da duna frontal perturbada pelo pisoteio, idêntico ao sugerido por Grime (1998) para as espécies subordinadas. O autor refere que - à semelhança do que acontece com M. marina, que chega a atapetar completamente a superfície (fig. 5) - estas espécies experimentam, logo a seguir a uma perturbação, o aumento temporário do seu vigor e taxa de cobertura. A rápida expansão desta última traz consequências, positivas e negativas, ao recrutamento dos propágulos de diferentes espécies no local. Assim, não são exclusivamente os atributos das espécies (em particular os que lhes conferem uma maior capacidade de regeneração e de sobrevivência) que comandam a recolonização de áreas perturbadas. Na realidade, Grime (1998) sugere que, devido à elevada taxa de cobertura que atingem em tão pouco tempo, as espécies subordinadas atuam como um filtro ambiental, inibindo o restabelecimento das plantas dominantes nos locais perturbados, ao mesmo tempo que favorecem o crescimento de outras espécies, habitualmente pouco abundantes. Isto significa, portanto, que as espécies subordinadas controlam a dominância e a diversidade específica que irá caracterizar as novas comunidades que se formam naqueles locais.

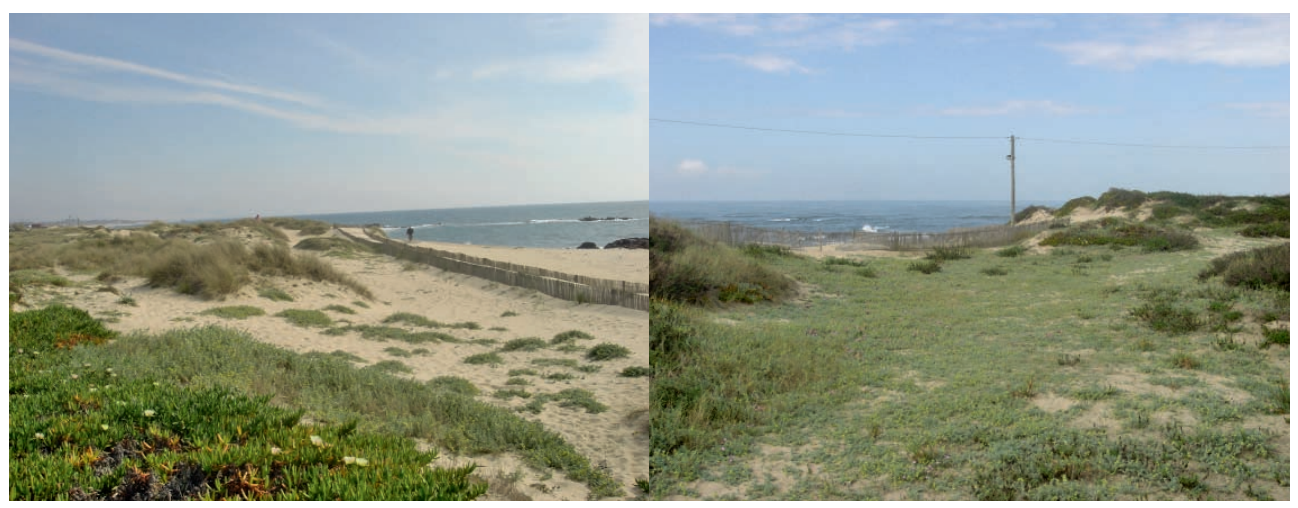

Fig. 5 - Predomínio de Medicago marina na recolonização inicial dos caminhos após o cessar do pisoteio, Vila Nova de Gaia, 2005.

Fig. 5 - Initial recolonization of sand paths by Medicago marina after trampling disturbance, Vila Nova de Gaia, 2005. (See coloured version online) 


\section{Consequências biogeomorfológicas da recolonização inicial dos caminhos por Medicago marina}

As observações de campo levadas a cabo entre 2005 e 2006, mostraram claramente que $M$. marina constitui a principal espécie (senão a única) responsável pela recolonização inicial dos caminhos na duna frontal em Vila Nova de Gaia, interditos à passagem de visitantes desde 2002-2003 (fig. 5). A extensa cobertura da superfície associada a esta planta (fomentada pelo crescimento de caules prostrados) diminui significativamente a velocidade do vento junto ao solo, reduzindo a deflação entre a vertente a barlavento e a crista da duna, e estabiliza eficazmente a areia. Ora, tal estabilização da areia limita a propagação clonal de $A$. arenaria (que necessita de uma deposição regular para o desenvolvimento ótimo de novos indivíduos), pelo que esta planta dominante é removida (ou seja, filtrada) do conjunto das espécies que podem ressurgir nos caminhos, ficando confinada aos fragmentos de habitat dunar existentes, onde mantém populações remanescentes. Em contrapartida, as condições biogeomorfológicas proporcionadas por M. marina "preparam" a chegada de uma nova vaga de propágulos, nomeadamente as sementes de uma diversidade de espécies anuais termófilas, apenas frequentes na vertente a sotavento, incapazes de sobreviver ao soterramento pela areia. Desta forma, assistiu-se a um aumento considerável da riqueza e abundância destas espécies - elas próprias acabam por substituir M. marina (observação pessoal em 2007 e 2008) -, nos primeiros anos após a intervenção da Câmara Municipal, contrariando a tendência das comunidades dunares não fragmentadas pelo pisoteio, onde $A$. arenaria é dominante, para se irem tornando monoespecíficas com o tempo.

É importante salientar que o filtro ambiental associado a M. marina resultou particularmente acentuado, tanto no tempo como no espaço, pelo facto da intervenção em causa ter passado pela colocação de armadilhas de areia (estacaria) ao longo de toda a frente dunar, que se encontrava, à data, bastante destruída pela deflação (e, eventualmente, pela erosão marinha). Esta medida veio estimular uma importante retenção de areia a barlavento da posição da crista da duna frontal, dando origem a um novo cordão dunar, em detrimento do robustecimento do já existente (fig. 5). Neste, dada a "passagem" de uma muito menor quantidade de areia transportada pelo vento, a rápida recolonização por M. marina foi fortemente incrementada, e - não obstante o aumento de biodiversidade aí observado -, as plantas mais favorecidas por esta nova situação têm como ação primordial a estabilização da areia, ou não desempenham qualquer função morfológica, sendo intolerantes ao soterramento; por consequência, todas as formas erosivas e deprimidas (0,5-1m de profundidade), correspondentes aos caminhos, se mantiveram. Sem o estímulo da acumulação regular e abundante de areia, a propagação e o crescimento vertical de $A$. arenaria foram seriamente comprometidos, tal como a reconstituição morfológica da duna frontal existente, que poderia ser sustentada por esta planta, caso as armadilhas de areia tivessem sido instaladas ao longo dos caminhos, desde a frente da duna até à crista, no sentido de promover a diferença em altura relativamente à praia alta - zona atingida pelo mar em situação de temporal e onde se começou a desenvolver um cordão dunar embrionário. 


\section{AGRADECIMENTOS}

Os autores agradecem aos revisores científicos a leitura cuidada e as sugestões formuladas que em muito contribuíram para melhorar este artigo.

Desejamos também manifestar o nosso agradecimento a Raquel Paixão que elaborou as figuras 1 e 3.

\section{BIBLIOGRAFIA}

Acosta A, Ercole S, Stanisci A, Pillar V, Blasi C (2007) Coastal vegetation zonation and dune morphology in some Mediterranean ecosystems. Journal of Coastal Research, 23: 1518-1524.

Araújo R, Honrado J, Granja H, Pinho S, Caldas F (2002) Vegetation complexes of coastal sand dunes as an evaluation instrument of geomorphologic changes in the coastline. In Gomes $\mathrm{F}$, Pinto F, Neves L (eds.) Littoral 2002: The changing coast, Associação EUROCOASTPortugal, Porto: 337-339.

Beisner B, Haydon D, Cuddington K (2003) Alternative stable states in ecology. Frontiers in Ecology and the Environment, 1: 376-382.

Bonanomi G, Incerti G, Mazzoleni S (2011) Assessing occurrence, specificity, and mechanisms of plant facilitation in terrestrial ecosystems. Plant Ecology, 212:1777-1790.

Bonanomi G, Rietkerk M, Dekker S, Mazzoleni S (2008) Islands of fertility induce co-occurring negative and positive plant-soil feedbacks promoting coexistence. Plant Ecology, 197: 207-218.

Cheplick G (2005) Patterns in the distribution of American beachgrass (Ammophila breviligulata) and the density and reproduction of annual plants on a coastal beach. Plant Ecology, 180: 57-67.

Clark C, Poulsen J, Levey D, Osenberg C (2007) Are plant populations seed limited? A critique and meta-analysis of seed addition experiments. The American Naturalist, 170: 128-142.

Dale M (1995) Evaluating classification strategies. Journal of Vegetation Science, 6: 437-440.

de Leo G, Levin S (1997) The multifaceted aspects of ecosystem integrity. Conservation Ecology, 1(3). http://www.consecol.org/vol1/iss1/ art3. [Acedido em 2 de dezembro de 2011].

Dech J, Maun M (2005) Zonation of vegetation along a burial gradient on the leeward slopes of Lake Huron sand dunes. Canadian Journal of Botany, 83: 227-236.
Denslow J (1985) Disturbance-mediated coexistence of species. In Pickett S, White $\mathrm{P}$ (Eds.) The ecology of natural disturbance and patch $d y$ namics. Academic Press, New York: 307-323.

Denslow J (1980) Patterns of plant species diversity during succession under different disturbance regimes. Oecologia, 46: 18-21.

Doust L (1981) Population dynamics and local specialization in a clonal perennial (Ranunculus repens): I. The dynamics of ramets in contrasting habitats. Journal of Ecology, 69: 743-755.

Eriksson O (1989) Seedling dynamics and life histories in clonal plants. Oikos, 55: 231-238.

Eriksson O, Ehrlén J (1992) Seed and microsite limitation of recruitment in plant populations. Oecologia, 91: 360-364.

Feinsinger P (2001) Designing field studies for biodiversity conservation. Island Press (The Nature Conservancy), Washington.

Forey E, Chapelet B, Vitasse Y, Tilquin M, Touzard B, Michalet R (2008) The relative importance of disturbance and environmental stress at local and regional scales in French coastal sand dunes. Journal of Vegetation Science, 19: 493-502.

Fortin M, Dale M, Hoef J (2002) Spatial analysis in ecology. In El-Shaarawi A, Piegorsch W (Eds.) Encyclopedia of Environmetrics. John Wiley \& Sons, Chichester: 2051-2058.

García-Mora M, Gallego-Fernández J, García-Novo F (1999) Plant functional types in coastal foredunes in relation to environmental stress and disturbance. Journal of Vegetation Science, 10: 27-34.

Gardner R, Engelhardt K (2008) Spatial processes that maintain biodiversity in plant communities. Perspectives in Plant Ecology, Evolution and Systematics, 9: 211-228.

Gauch H, Whittaker H (1981) Hierarchical classification of community data. Journal of Ecology, 69: 537-557. 
Gilbert M, Pammenter N, Ripley B (2008) The growth responses of coastal dune species are determined by nutrient limitation and sand burial. Oecologia, 156: 169-178.

Gomes A A, Ramos-Pereira A, Araújo M A, Sousa A, Veloso-Gomes F (2006) Douro's Estuary Dynamics. In Ramos Pereira A, and Collaboraters (Eds.) Portugal: coastal dynamics. Publicações da Associação Portuguesa de Geomorfólogos, APGeom, Lisboa, IV: 25-29.

Greig-Smith P (1961) Data on pattern within plant communities: II. Ammophila arenaria (L.) Link. The Journal of Ecology, 49: 703-708.

Grime J (1998) Benefits of plant diversity to ecosystems: immediate, filter and founder effects. Journal of Ecology, 86: 902-910.

Hesp P (2002) Foredunes and blowouts: initiation, geomorphology and dynamics. Geomorphology, 48: 245-268.

Hill M (1979) TWINSPAN: a FORTRAN Program for Arranging Multivariate Data in an Ordered Two-Way Table by Classification of the Individuals and Attributes. Cornell University, New York.

Honrado J, Vicente J, Lomba A, Alves P, Macedo J, Henriques R, Granja H, Caldas F (2010) Fine-scale patterns of vegetation assembly in the monitoring of changes in coastal sand-dune landscapes. Web Ecology, 10:1-14.

Hook P, Lauenroth W, Burke I (1994) Spatial patterns of roots in a semiarid grassland: abundance of canopy openings and regeneration gaps. Journal of Ecology, 82: 485-494.

Jentsch A (2007) The challenge to restore processes in face of nonlinear dynamics: on the crucial role of disturbance regimes. Restoration Ecology, 15: 334-339.

Jones C, Lawton J, Shachak M (1997) Positive and negative effects of organisms as physical ecosystem engineers. Ecology, 78: 1946-1957.

Jones C, Lawton J, Shachak M (1994) Organisms as ecosystem engineers. Oikos, 69: 373-386.

Keddy P (1992) Assembly and response rules: two goals for predictive community ecology. Journal of Vegetation Science, 3: 157-164.

Kent M, Owen N, Dale P, Newnham R, Giles T (2001) Studies of vegetation burial: a focus for biogeography and biogeomorphology? Progress in Physical Geography, 25: 455-482.

Kotanen P (1997) Effects of gap area and shape on recolonization by grassland plants with differing reproductive strategies. Canadian Journal of Botany, 75: 352-361.
Krebs C (2001) Ecology: the experimental analysis of distribution and abundance. Benjamin Cummings, San Francisco.

Laranjeira M (2010) Fragmentação pelo pisoteio dos sistemas de duna frontal: casos de Vila Nova de Gaia. Dissertação de doutoramento, Universidade do Minho, Braga.

Levin N, Kidron G, Ben-Dor E (2008) A field quantification of coastal dune perennial plants as indicators of surface stability, erosion or deposition. Sedimentology, 55: 751-772.

Lomba A, Alves P, Honrado J (2008) Endemic sand dune vegetation of the Northwest Iberian Peninsula: diversity, dynamics, and significance for bioindication and monitoring of coastal landscapes. Journal of Coastal Research, 24:113-121.

Łaska G (2001) The disturbance and vegetation dynamics: a review and an alternative framework. Plant Ecology, 157: 77-99.

Maun M (1998) Adaptations of plants to burial in coastal sand dunes. Canadian Journal of Botany, 76: 713-738.

Moreno-Casasola P (1986) Sand movement as a factor in the distribution of plant communities in a coastal dune system. Vegetatio, 65: 67-76.

Packham J, Willis A (1997) Ecology of dunes, salt marsh and shingle. Chapman \& Hall, London.

Pemadasa M, Greig-Smith P, Lovell P (1974) A quantitative description of the distribution of annuals in the dune system at Aberffraw, Anglesey. The Journal of Ecology, 62: 379-402.

Perry G (2002) Landscapes, space and equilibrium: shifting viewpoints. Progress in Physical Geography, 26: 339-359.

Ramos-Pereira A (2004) A faixa litoral. In Feio M, Daveau S (Eds.) O relevo de Portugal. Grandes unidades regionais. Publicações da Associação Portuguesa de Geomorfólogos, APGeom, Coimbra, IV: 133-147.

Ramos-Pereira A, Trindade J, Neves M (2006) The portuguese littoral setting. In Ramos-Pereira A and collaborators (Eds.) Portugal: coastal dynamics. Publicações da Associação Portuguesa de Geomorfólogos, APGeom, Lisboa, IV: 13-18

Ranwell D (1958) Movement of vegetated sand dunes at Newborough Warren, Anglesey. The Journal of Ecology, 46: 83-100.

Reid M, Thompson S (1996) Ecological fieldwork methods. In Watts S, Halliwell L (Eds.) Essential Environmental Science: Methods \& Techniques. Routledge, London and New York: 351-388. 
Smith R, Smith T (2001) Ecology and field biology. Benjamin Cummings, San Francisco.

Stallins J (2002) Dune plant species diversity and function in two barrier island biogeomorphic systems. Plant Ecology, 165: 183-196.

Temperton V, Hobbs R, Nuttle T, Halle S (Eds.) (2004) Assembly rules and restoration ecology: bridging the gap between theory and practice. Island Press, Washington.

Townend J (2002) Practical statistics for environmental and biological scientists. John Wiley \& Sons, Chichester.

Tzatzanis M, Wrbka T, Sauberer N (2003) Landscape and vegetation responses to human impact in sandy coasts of Western Crete, Greece. Journal for Nature Conservation, 11: 187-195.

Urban D (2003) Spatial analysis in ecology: mantel's test. University of California, St. ${ }^{a}$ Barbara. http://www.nceas.ucsb.edu/scicomp/Dloads/SpatialAnalysisEcologist/SpatialEcology/ MantelTest.pdf [Acedido em 26 de maio de 2009]

Vandvik V (2004) Gap dynamics in perennial subalpine grasslands: trends and processes change during secondary succession. Journal of Ecology, 92: 86-96.

Vermeersch S, Genst W, Vermoesen F, Triest L (2003) The influence of transformations of an ordinal scale of a floristic gradient, applied on a TWINSPAN classification. Flora, 198: 389-403.

Walker L, del Moral R (2003) Primary succession and ecosystem rehabilitation. Cambridge University Press, Cambridge.

Watkinson A (1990) The population dynamics of Vulpia fasciculata: a nine-year study. Journal of Ecology, 78: 196-209.

White P, Jentsch A (2004) Disturbance, succession, and community assembly in terrestrial plant communities. In Temperton V, Hobbs R, Nuttle T, Halle $\mathrm{S}$ (Eds.) Assembly rules and restoration ecology: bridging the gap between theory and practice. Island Press, Washington: 342-366.

White P, Pickett S (1985) Natural disturbance and patch dynamics: an introduction. In Pickett $\mathrm{S}$, White P (eds.) The ecology of natural disturbance and patch dynamics. Academic Press, New York: 3-13.

Willis A, Folkes B, Hope-Simpson J, Yemm E (1959) Braunton Burrows: the dune system and its vegetation. Part II. The Journal of Ecology, 47: 249-288.

Young T, Chase J, Huddleston R (2001) Community succession and assembly: comparing, contrasting and combining paradigms in the context of ecological restoration. Ecological Restoration, 19: 5-18.

As autoras deste texto escrevem segundo o novo acordo ortográfico 